\title{
Boundary regularity for $p$-harmonic functions and solutions of the obstacle problem on metric spaces
}

\author{
By Anders BJörN and Jana BJöRN
}

(Received Jun. 6, 2005)

(Revised Jan. 5, 2006)

\begin{abstract}
We study $p$-harmonic functions in complete metric spaces equipped with a doubling Borel measure supporting a weak $(1, p)$-Poincaré inequality, $1<$ $p<\infty$. We establish the barrier classification of regular boundary points from which it also follows that regularity is a local property of the boundary. We also prove boundary regularity at the fixed (given) boundary for solutions of the onesided obstacle problem on bounded open sets. Regularity is further characterized in several other ways.

Our results apply also to Cheeger $p$-harmonic functions and in the Euclidean setting to $\mathscr{A}$-harmonic functions, with the usual assumptions on $\mathscr{A}$.
\end{abstract}

\section{Introduction.}

Let $\Omega \subset \boldsymbol{R}^{n}$ be a nonempty bounded open set and let $f \in C(\partial \Omega)$. Then the Perron method provides a unique solution $u$ of the Dirichlet problem (the boundary value problem) for the Laplace equation, i.e. $u$ is harmonic in $\Omega$ and takes the boundary values $f$ in a weak sense. A point $x_{0} \in \partial \Omega$ is said to be regular if $\lim _{\Omega \ni y \rightarrow x_{0}} u(y)=f\left(x_{0}\right)$ for every $f \in C(\partial \Omega)$. Wiener [35] characterized regular boundary points by the so called Wiener criterion in 1924. The same year Lebesgue [27] characterized regular boundary points in terms of barriers.

One can also consider the corresponding problem for $p$-harmonic functions, $1<p<$ $\infty$. This leads to a nonlinear theory, and a similar characterization has been proved, see Heinonen-Kilpeläinen-Martio [14], Kilpeläinen-Malý [19], Maz'ya [28] and Mikkonen $[\mathbf{2 9}]$.

More recently, potential theory has been developed in complete metric spaces equipped with a doubling measure supporting a Poincaré inequality. From the results in Björn-MacManus-Shanmugalingam [8] and Björn-Björn-Shanmugalingam [4] it follows that the Wiener criterion is sufficient for regularity, under the additional assumption that the space is linearly locally connected, see Remark 7.4. In this paper we prove that the barrier characterization holds in metric spaces, from which it follows that regularity is a local property of the boundary. Several other characterizations of regularity are also given, see Theorem 6.1 .

Instead of just studying the Dirichlet problem for $p$-harmonic functions we study the associated (one-sided) obstacle problem with a given obstacle $\psi$ and a given boundary value function $f$. If $\psi \equiv-\infty$ the obstacle problem reduces to the usual Dirichlet problem.

2000 Mathematics Subject Classification. Primary 35J65; Secondary 31C45, 35B65, 46E35, 49N60.

Key Words and Phrases. barrier, doubling, metric space, nonlinear, obstacle problem, $p$-harmonic, Poincaré inequality, regular, superharmonic. 
We show in Section 5 that if $x_{0}$ is a regular boundary point and $f$ is continuous at $x_{0}$, then the solution of the obstacle problem takes the boundary value $f\left(x_{0}\right)$ (in the classical sense) if and only if ess lim $\sup _{\Omega \ni y \rightarrow x_{0}} \psi(y) \leq f\left(x_{0}\right)$. (We also provide an example showing that the latter condition is violated for some soluble obstacle problems.)

For Sobolev extendable boundary data $f$, the one-sided obstacle problem can be considered as a special case of the double obstacle problem (putting $\psi_{1}=\psi_{2}=f$ outside $\Omega$ ). In the Euclidean case, this can be used to obtain special cases of some of the results in Section 5 from the free boundary regularity results for the double obstacle problem in Dal Maso-Mosco-Vivaldi [9] $(p=2)$ and Kilpeläinen-Ziemer [20].

There are many different examples of metric spaces equipped with a doubling measure satisfying a Poincaré inequality. Here are some of them:

(1) Unweighted and weighted Euclidean spaces, see the monograph by HeinonenKilpeläinen-Martio [14].

(2) Riemannian manifolds with nonnegative Ricci curvature satisfy the (1,2)-Poincaré inequality, see Saloff-Coste [30].

(3) Graphs, see Shanmugalingam [33].

(4) The Heisenberg group $\boldsymbol{H}_{1}=\boldsymbol{C} \times \boldsymbol{R}$ with the Lebesgue measure and the metric

$$
d\left((z, t),\left(z^{\prime}, t^{\prime}\right)\right)=\left(\left|z-z^{\prime}\right|^{4}+\left(t-t^{\prime}+2 \operatorname{Im} \bar{z} z^{\prime}\right)^{2}\right)^{1 / 4}
$$

satisfies the (1,1)-Poincaré inequality, see Heinonen [13], Theorem 9.27. Note that $\boldsymbol{H}_{1}$ is topologically 3 -dimensional but Ahlfors 4-regular, i.e. $\mu(B) \approx(\operatorname{diam} B)^{4}$ for balls $B$.

(5) For every $Q>1$, Laakso [26] showed that there is an Ahlfors $Q$-regular space satisfying the $(1,1)$-Poincaré inequality.

(6) In A. Björn [2], an example is constructed where a line (with a one-dimensional measure) is glued to a triangle (with a two-dimensional measure) so that the union satisfies the (1,1)-Poincaré inequality.

The results and proofs given in this paper also hold for Cheeger $p$-harmonic functions, see, e.g., Björn-Björn-Shanmugalingam [5] for a discussion about them. The results and proofs also hold for $\mathscr{A}$-harmonic functions as defined on p. 57 of Heinonen-KilpeläinenMartio [14], assuming that $\mathscr{A}$ satisfies the degenerate ellipticity conditions (3.3)-(3.7) on p. 56 of $[\mathbf{1 4}]$.

The outline of the paper is as follows. In Section 2, we define Newtonian spaces, the Sobolev type spaces considered in metric spaces, and give some of their properties. In Section 3, we define $p$-harmonic functions, $p$-superharmonic functions and the obstacle problem, and the basic theory is also explained. In Section 4, we characterize regular boundary points using barriers. Boundary regularity for the obstacle problem is studied in Section 5. This is then used in the following section to give several other characterizations of regular boundary points.

We end the paper, in Section 7, with a quantitative estimate for the solution of the obstacle problem near a regular boundary point, and the sufficiency of the Wiener criterion when $X$ is linearly locally connected. 
ACKNowledgement. Both authors were supported by the Swedish Research Council and Gustaf Sigurd Magnuson's fund of the Royal Swedish Academy of Sciences. This research started while they were visiting the Department of Mathematical Analysis at the Charles University in Prague during the autumn 2003.

We wish to thank Hiroaki Aikawa and Nageswari Shanmugalingam for comments on early versions of this paper.

\section{Notation and preliminaries.}

We assume throughout the paper that $X=(X, d, \mu)$ is a complete metric space endowed with a metric $d$ and a positive complete Borel measure $\mu$ such that $0<\mu(B)<$ $\infty$ for all balls $B \subset X$ (we make the convention that balls are nonempty and open). It is more or less immediate that $\mu$ is a Borel regular measure, and we emphasize that the $\sigma$-algebra on which $\mu$ is defined is obtained by completion of the Borel $\sigma$-algebra. We also assume that $1<p<\infty$. (In the beginning of Section 3 we make some further assumptions on $X$ that are assumed in the rest of the paper.)

The measure $\mu$ is doubling if there exists a constant $C>0$ such that for all balls $B=B\left(x_{0}, r\right):=\left\{x \in X: d\left(x, x_{0}\right)<r\right\}$ in $X$,

$$
\mu(2 B) \leq C \mu(B)
$$

where $\lambda B=B\left(x_{0}, \lambda r\right)$.

In this paper a curve in $X$ is a nonconstant continuous mapping from a compact interval, which is rectifiable. A curve can thus be parameterized by arc length $d s$.

DeFinition 2.1. A nonnegative Borel function $g$ on $X$ is an upper gradient of an extended real-valued function $f$ on $X$ if for all curves $\gamma:\left[0, l_{\gamma}\right] \rightarrow X$,

$$
\left|f(\gamma(0))-f\left(\gamma\left(l_{\gamma}\right)\right)\right| \leq \int_{\gamma} g d s
$$

whenever both $f(\gamma(0))$ and $f\left(\gamma\left(l_{\gamma}\right)\right)$ are finite, and $\int_{\gamma} g d s=\infty$ otherwise. If $g$ is a nonnegative measurable function on $X$ and if (2.1) holds for $p$-almost every curve, then $g$ is a $p$-weak upper gradient of $f$.

By saying that (2.1) holds for $p$-almost every curve we mean that it fails only for a curve family with zero $p$-modulus, see Definition 2.1 in Shanmugalingam [31]. It is implicitly assumed that $\int_{\gamma} g d s$ is defined (with a value in $[0, \infty]$ ) for $p$-almost every curve.

If $g \in L^{p}(X)$ is a $p$-weak upper gradient of $f$, then one can find a sequence $\left\{g_{j}\right\}_{j=1}^{\infty}$ of upper gradients of $f$ such that $g_{j} \rightarrow g$ in $L^{p}(X)$, see Lemma 2.4 in KoskelaMacManus [25].

If $f$ has an upper gradient in $L^{p}(X)$, then it has a minimal $p$-weak upper gradient $g_{f} \in L^{p}(X)$ in the sense that for every $p$-weak upper gradient $g \in L^{p}(X)$ of $f, g_{f} \leq g$ $\mu$-a.e., see Corollary 3.7 in Shanmugalingam [32].

So far in the literature it has most often been assumed (at least implicitly) that 
$p$-weak upper gradients are Borel functions. However, it was observed by Heinonen (see Heinonen-Koskela-Shanmugalingam-Tyson [15] and Shanmugalingam [34]), that this leads to a problem in pasting formulas, e.g. if $g_{u}$ and $g_{v}$ are Borel minimal $p$-weak upper gradients of $u, v \in N^{1, p}(X)$, then $g=g_{u} \chi_{\{u>v\}}+g_{v} \chi_{\{v \geq u\}}$ is not in general a Borel function, and hence not in general a Borel $p$-weak upper gradient of $\max \{u, v\}$. However, $g$ is a $p$-weak upper gradient of $\max \{u, v\}$ with our definition, and in fact is also minimal. Similarly $g_{u} \chi_{\{u<v\}}+g_{v} \chi_{\{v \leq u\}}$ is a minimal $p$-weak upper gradient of $\min \{u, v\}$. For proofs of these facts see Section 3 in the preprint version of this paper $[3]$.

Definition 2.2. We say that $X$ supports a weak $(1, q)$-Poincaré inequality if there exist constants $C>0$ and $\lambda \geq 1$ such that for all balls $B \subset X$, all measurable functions $f$ on $X$ and all upper gradients $g$ of $f$,

$$
f_{B}\left|f-f_{B}\right| d \mu \leq C(\operatorname{diam} B)\left(f_{\lambda B} g^{q} d \mu\right)^{1 / q}
$$

where $f_{B}:=f_{B} f d \mu:=\int_{B} f d \mu / \mu(B)$. If $\lambda=1$, then $X$ supports a $(1, q)$-Poincaré inequality.

By the Hölder inequality it is easy to see that if $X$ supports a weak $(1, q)$-Poincaré inequality, then it supports a weak $(1, s)$-Poincaré inequality for every $s>q$. In the above definition of Poincaré inequality we can equivalently assume that $g$ is a $q$-weak upper gradient-see the comments above.

If $X$ is complete then it is equivalent to require that (2.2) holds for all $f \in \operatorname{Lip}_{c}(X)$ and all upper gradients $g \in \operatorname{Lip}_{c}(X)$ of $f$, see Keith [16], Theorem 2. Here $\operatorname{Lip}_{c}(A)=$ $\{f \in \operatorname{Lip}(A): \operatorname{supp} f \Subset A\}$.

Following Shanmugalingam [31], we define a version of Sobolev spaces on the metric space $X$.

Definition 2.3. Whenever $u \in L^{p}(X)$, let

$$
\|u\|_{N^{1, p}(X)}=\left(\int_{X}|u|^{p} d \mu+\inf _{g} \int_{X} g^{p} d \mu\right)^{1 / p},
$$

where the infimum is taken over all upper gradients of $u$. The Newtonian space on $X$ is the quotient space

$$
N^{1, p}(X)=\left\{u:\|u\|_{N^{1, p}(X)}<\infty\right\} / \sim,
$$

where $u \sim v$ if and only if $\|u-v\|_{N^{1, p}(X)}=0$.

The space $N^{1, p}(X)$ is a Banach space and a lattice, see Shanmugalingam [31].

Definition 2.4. The $p$-capacity of a set $E \subset X$ is the number

$$
C_{p}(E)=\inf \|u\|_{N^{1, p}(X)}^{p},
$$


where the infimum is taken over all $u \in N^{1, p}(X)$ such that $u=1$ on $E$.

The $p$-capacity is countably subadditive. For this and other properties as well as equivalent definitions of the $p$-capacity we refer to Kilpeläinen-Kinnunen-Martio [18] and Kinnunen-Martio [21], [22].

We say that a property regarding points in $X$ holds $p$-quasieverywhere (p-q.e.) if the set of points for which the property does not hold has $p$-capacity zero. The $p$-capacity is the correct gauge for distinguishing between two Newtonian functions. If $u \in N^{1, p}(X)$, then $u \sim v$ if and only if $u=v p$-q.e. Moreover, Corollary 3.3 in Shanmugalingam [31] shows that if $u, v \in N^{1, p}(X)$ and $u=v \mu$-a.e., then $u \sim v$.

Further, if $X$ supports a weak $(1, p)$-Poincaré inequality and $\mu$ is doubling, then Lipschitz functions are dense in $N^{1, p}(X)$ and the functions in $N^{1, p}(X)$ are $p$-quasicontinuous, see [31] and Björn-Björn-Shanmugalingam [6]. This means that in the Euclidean setting, $N^{1, p}\left(\boldsymbol{R}^{n}\right)$ is the refined Sobolev space as defined on p. 96 of Heinonen-KilpeläinenMartio [14].

To be able to compare the boundary values of Newtonian functions we need a Newtonian space with zero boundary values.

Definition 2.5. For open sets $A, E \subset X$, we introduce the space of Newtonian functions with zero values in $A \backslash E$ as follows,

$$
N_{0}^{1, p}(E ; A)=\left\{\left.f\right|_{E \cap A}: f \in N^{1, p}(A) \text { and } f=0 \text { in } A \backslash E\right\} .
$$

We also let $N_{0}^{1, p}(E)=N_{0}^{1, p}(E ; X)$.

One can replace the assumption " $f=0$ in $A \backslash E$ " with " $f=0$-q.e. in $A \backslash E$ " without changing the obtained space $N_{0}^{1, p}(E ; A)$. It is also quite easy to see that $N_{0}^{1, p}(E ; A)=$ $N_{0}^{1, p}(E ; A \cap \bar{E})$, the ideas for a proof can be found in the proof of Lemma 5.3. Note also that if $C_{p}(A \backslash E)=0$, then $N_{0}^{1, p}(E ; A)=N^{1, p}(E \cap A)$.

We end this section by recalling some standard notation. We let $f_{+}=\max \{f, 0\}$ and $f_{-}=\max \{-f, 0\}$. By a continuous function we always mean a real-valued continuous function, whereas a semicontinuous function is allowed to be extended real-valued, i.e. to take values in the extended real line $\overline{\boldsymbol{R}}:=[-\infty, \infty]$.

Unless otherwise stated, the letter $C$ denotes various positive constants whose exact values are unimportant and may vary with each usage.

\section{The obstacle problem and $p$-harmonic functions.}

From now on we assume that $X$ is complete, and that $\mu$ is doubling and supports a weak $(1, p)$-Poincaré inequality. Note that some authors assume that $X$ is proper rather than complete, but, since $\mu$ is doubling, $X$ is proper if and only if $X$ is complete. By Keith-Zhong [17] it follows that $X$ supports a weak $(1, q)$-Poincaré inequality for some $q \in[1, p)$, which was earlier a standard assumption. We also assume throughout the rest of this paper that $\Omega \subset X$ is a nonempty bounded open set in $X$ such that $C_{p}(X \backslash \Omega)>0$. (If $X$ is unbounded then the condition $C_{p}(X \backslash \Omega)>0$ is of course immediately fulfilled.)

We follow Kinnunen-Martio [23] making the following definition of the obstacle problem. 
Definition 3.1. Let $V \subset X$ be a nonempty bounded open set with $C_{p}(X \backslash V)>0$. Let $f \in N^{1, p}(V)$ and $\psi: V \rightarrow \overline{\boldsymbol{R}}$. Then we define

$$
\mathscr{K}_{\psi, f}(V)=\left\{v \in N^{1, p}(V): v-f \in N_{0}^{1, p}(V) \text { and } v \geq \psi \mu \text {-a.e. in } V\right\} \text {. }
$$

Furthermore, a function $u \in \mathscr{K}_{\psi, f}(V)$ is a solution of the $\mathscr{K}_{\psi, f}(V)$-obstacle problem if

$$
\int_{V} g_{u}^{p} d \mu \leq \int_{V} g_{v}^{p} d \mu \quad \text { for all } v \in \mathscr{K}_{\psi, f}(V)
$$

Kinnunen-Martio [23], Theorem 3.2, showed that if $\mathscr{K}_{\psi, f}(V) \neq \varnothing$, then there is a solution of the $\mathscr{K}_{\psi, f}(V)$-obstacle problem, and this solution is unique up to equivalence in $N^{1, p}(V)$. They also showed, Theorem 5.1 in [23], that if $u$ is a solution then its lower semicontinuous regularization $u^{*}(x)=\operatorname{ess}_{\liminf } \operatorname{lix}_{y \rightarrow x} u(y)$ is also a solution and this solution is the unique lower semicontinuously regularized solution. Furthermore, $u^{*}$ is $p$-superharmonic (see Definition 3.3). If the obstacle $\psi$ is continuous they showed that $u^{*}$ is also continuous, see Theorem 5.5 in [23]. They actually considered continuous functions which are even allowed to take the value $-\infty$. We will need the following special case of their result. For $f \in N^{1, p}(V)$, define $H_{V} f$ to be the continuous solution of the $\mathscr{K}_{-\infty, f}(V)$-obstacle problem. If $V=\Omega$, we usually suppress the index and merely write $H f=H_{\Omega} f$ and $\mathscr{K}_{\psi, f}=\mathscr{K}_{\psi, f}(\Omega)$.

Proposition 3.2. Let $f \in N^{1, p}(\Omega)$ be continuous. Then there is a continuous

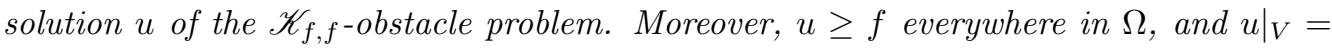
$H_{V} f$ for the open set $V=\{x \in \Omega: u(x)>f(x)\}$.

Proof. Observe first that $f \in \mathscr{K}_{f, f}$. That there is a continuous solution of

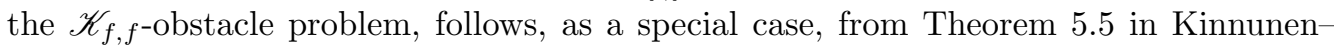
Martio [23]. The conclusion that $\left.u\right|_{V}=H_{V} f$ was observed in the proof of Theorem 7.7 in [23]. That $u \geq f$ everywhere in $\Omega$ follows directly from the assumption that $u \geq f$ $\mu$-a.e. in $\Omega$ together with the continuity of $u$ and $f$.

A function $u$ is a $p$-superminimizer if it is a solution of the $\mathscr{K}_{u, u}\left(\Omega^{\prime}\right)$-obstacle problem for every subdomain $\Omega^{\prime} \Subset \Omega$. A solution $u$ to the $\mathscr{K}_{\psi, f}$-obstacle problem is a $p$-superminimizer. Conversely, if $u \in N^{1, p}(\Omega)$ is a $p$-superminimizer, then $u$ is a solution of the $\mathscr{K}_{u, u}(\Omega)$-obstacle problem. A function $u$ is a $p$-minimizer if it is a solution of the $\mathscr{K}_{-\infty, u}\left(\Omega^{\prime}\right)$-obstacle problem for every subdomain $\Omega^{\prime} \Subset \Omega$, or equivalently if both $u$ and $-u$ are $p$-superminimizers.

By Proposition 3.8 and Corollary 5.5 in Kinnunen-Shanmugalingam [24], a $p$ minimizer can be modified on a set of $p$-capacity zero so that it becomes locally Hölder continuous in $\Omega$. A p-harmonic function is a continuous $p$-minimizer. By Corollary 6.4 in [24], $p$-harmonic functions $u$ satisfy the strong maximum principle: If $u$ attains its minimum or maximum in some component $G$ of $\Omega$, then $\left.u\right|_{G}$ is constant. The sum of two $p$-harmonic functions is, in general, not a $p$-harmonic function. Nevertheless, if $u$ is $p$-harmonic and $\alpha, \beta \in \boldsymbol{R}$, then $\alpha u+\beta$ is also $p$-harmonic.

If $f_{1}, f_{2} \in N^{1, p}(\Omega)$ and $\left(f_{1}-f_{2}\right)_{+} \in N_{0}^{1, p}(\Omega)$, then $H f_{1} \leq H f_{2}$ in $\Omega$. (This is a 
special case of Lemma 5.4.) It follows that for $f \in N^{1, p}(\bar{\Omega}), H f$ only depends on $\left.f\right|_{\partial \Omega}$. A Lipschitz function $f$ on $\partial \Omega$ can be extended to a function $\tilde{f} \in \operatorname{Lip}(\bar{\Omega})$ such that $f=\tilde{f}$ on $\partial \Omega$. As $H \tilde{f}$ does not depend on the choice of extension, we define $H f=H \tilde{f}$.

Definition 3.3. A function $u: \Omega \rightarrow(-\infty, \infty]$ is $p$-superharmonic in $\Omega$ if

(i) $u$ is lower semicontinuous;

(ii) $u$ is not identically $\infty$ in any component of $\Omega$;

(iii) for every nonempty open set $\Omega^{\prime} \Subset \Omega$ and all functions $v \in \operatorname{Lip}\left(\partial \Omega^{\prime}\right)$, we have $H_{\Omega^{\prime}} v \leq u$ in $\Omega^{\prime}$ whenever $v \leq u$ on $\partial \Omega^{\prime}$.

A function $u: \Omega \rightarrow[-\infty, \infty)$ is $p$-subharmonic if $-u$ is $p$-superharmonic.

This definition is equivalent to the definition given in Kinnunen-Martio [23], Section 7, see A. Björn [2], Theorem 6.1. If $u$ and $v$ are $p$-superharmonic, $\alpha \geq 0$ and $\beta \in \boldsymbol{R}$, then $\alpha u+\beta$ and $\min \{u, v\}$ are $p$-superharmonic, but in general $u+v$ is not $p$ superharmonic. A $p$-superharmonic function is automatically lower semicontinuously regularized, see [23], Theorem 7.14. Moreover, a function $u \in N^{1, p}(\Omega)$ is $p$-superharmonic if and only if it is a lower semicontinuously regularized $p$-superminimizer, which happens if and only if $u$ is the lower semicontinuously regularized solution of the $\mathscr{K}_{u, u}$-obstacle problem. Note however that not all $p$-superharmonic functions belong to $N^{1, p}(\Omega)$, or even $N_{\text {loc }}^{1, p}(\Omega)$, and are therefore not $p$-superminimizers.

It follows from Lemma 7.11 in $[\mathbf{2 3}]$, that a $p$-superharmonic function $u$ satisfies the strong minimum principle: If $u$ attains its minimum in some component $G$ of $\Omega$, then $\left.u\right|_{G}$ is constant.

Definition 3.4. Given a function $f: \partial \Omega \rightarrow \overline{\boldsymbol{R}}$, let $\mathscr{U}_{f}$ be the set of all $p$-superharmonic functions $u$ on $\Omega$ bounded below such that

$$
\liminf _{\Omega \ni y \rightarrow x} u(y) \geq f(x) \quad \text { for all } x \in \partial \Omega
$$

Define the upper Perron solution of $f$ by

$$
\bar{P} f(x)=\inf _{u \in \mathscr{U}_{f}} u(x), \quad x \in \Omega
$$

Similarly, let $\mathscr{L}_{f}$ be the set of all $p$-subharmonic functions $u$ on $\Omega$ bounded above such that

$$
\limsup _{\Omega \ni y \rightarrow x} u(y) \leq f(x) \quad \text { for all } x \in \partial \Omega,
$$

and define the lower Perron solution of $f$ by

$$
\underline{P} f(x)=\sup _{u \in \mathscr{L}_{f}} u(x), \quad x \in \Omega
$$

If $\bar{P} f=\underline{P} f$, then we let $P_{\Omega} f=P f:=\bar{P} f$, and $f$ is said to be resolutive. 
The comparison principle given by Kinnunen-Martio [23], Theorem 7.2, shows that $\underline{P} f \leq \bar{P} f$ for all functions $f$.

We have now two ways of solving the Dirichlet problem for $p$-harmonic functions. We need the following results from Björn-Björn-Shanmugalingam [5].

Theorem 3.5 (Theorem 6.1 in $[\mathbf{5}]$ ). Let $f \in C(\partial \Omega)$. Then $f$ is resolutive.

Theorem 3.6 (Theorem 5.1 in $[\mathbf{5}]$ ). Let $f \in N^{1, p}(X)$. Then $P f=H f$ in $\Omega$.

Note that since $H f$ is independent of which representative of $f$ in $N^{1, p}(X)$ we pick, also $P f$ is independent of the representative.

Definition 3.7. A point $x_{0} \in \partial \Omega$ is regular if

$$
\lim _{\Omega \ni y \rightarrow x_{0}} \operatorname{Pf}(y)=f\left(x_{0}\right) \quad \text { for all } f \in C(\partial \Omega) .
$$

If $x_{0} \in \partial \Omega$ is not regular, then it is irregular. The set $\Omega$ is regular if every $x_{0} \in \partial \Omega$ is regular.

(See Theorem 6.1 for characterizations of regularity.)

Recall the following result from Björn-Björn-Shanmugalingam [4], Theorem 3.9.

Theorem 3.8 (The Kellogg property). The set of all irregular points on $\partial \Omega$ has p-capacity zero.

\section{Barrier characterization of regular points.}

Definition 4.1. A function $u$ is a barrier (with respect to $\Omega$ ) at $x_{0} \in \partial \Omega$ if

(i) $u$ is $p$-superharmonic in $\Omega$;

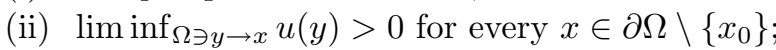

(iii) $\lim _{\Omega \ni y \rightarrow x_{0}} u(y)=0$.

By the strong minimum principle a barrier is always nonnegative. Moreover, a barrier is positive if every component $G \subset \Omega$ has a boundary point in $\partial G \backslash\left\{x_{0}\right\}$. The zero function is a barrier if and only if $\partial \Omega=\left\{x_{0}\right\}$.

TheOREM 4.2. Let $x_{0} \in \partial \Omega$ and $d(x):=d\left(x, x_{0}\right)$. Then the following are equivalent:

(a) The point $x_{0}$ is a regular boundary point.

(b) There is a barrier at $x_{0}$.

(c) There is a positive continuous barrier at $x_{0}$.

(d) It is true that

$$
\lim _{\Omega \ni y \rightarrow x_{0}} \operatorname{Pd}(y)=0 .
$$

(e) It is true that 


$$
\lim _{\Omega \ni y \rightarrow x_{0}} \bar{P} f(y)=f\left(x_{0}\right)
$$

for all bounded $f: \partial \Omega \rightarrow \boldsymbol{R}$ which are continuous at $x_{0}$.

The implication (a) $\Rightarrow$ (e) was actually obtained in Björn-Björn-Shanmugalingam [5], Corollary 7.2. In Section 6, we will give several other characterizations of regular boundary points by means of $p$-superharmonic functions and obstacle problems, see Theorem 6.1.

In order to prove Theorem 4.2 we will need the following lemma.

Lemma 4.3. Let $\Omega \subset X$ be a nonempty open set such that $C_{p}(X \backslash \Omega)>0$, then $C_{p}(\partial \Omega)>0$. Equivalently, if $X \backslash \bar{\Omega} \neq \varnothing$, then $C_{p}(\partial \Omega)>0$.

This is well known to the people working in the field. However, we have not found a good reference to this result, and therefore provide a proof. (A related result was given in Lemma 8.6 in Björn-Björn-Shanmugalingam [5].)

Proof. Note first that, since $X \backslash \bar{\Omega}$ is open, the following are equivalent:

(i) $X \backslash \bar{\Omega} \neq \varnothing$;

(ii) $\mu(X \backslash \bar{\Omega})>0$;

(iii) $C_{p}(X \backslash \bar{\Omega})>0$.

Assume that $C_{p}(\partial \Omega)=0$ and let $f=\chi_{\Omega}, g=0$ and $\Gamma_{\partial \Omega}$ be the family of curves $\gamma$ such that $\gamma \cap \partial \Omega \neq \varnothing$. By Lemma 3.6 in Shanmugalingam $[\mathbf{3 1}], \operatorname{Mod}_{p}\left(\Gamma_{\partial \Omega}\right)=0$. It follows that $g$ is a $p$-weak upper gradient of $f$. Now if there exists a point $x \in X \backslash \bar{\Omega}$ and $r$ is so large that $B(x, r) \cap \Omega \neq \varnothing$, then the pair $(f, g)$ violates the Poincaré inequality on $B(x, r)$. Hence, $X \backslash \bar{\Omega}=\varnothing$, and $C_{p}(X \backslash \Omega)=C_{p}(\partial \Omega)=0$.

Proof of Theorem 4.2. (a) $\Rightarrow$ (d) This is trivial.

(d) $\Rightarrow$ (e) Let $M=\sup _{\Omega}|f|$ and $\varepsilon>0$. Let further $r>0$ be such that $\mid f(x)-$ $f\left(x_{0}\right) \mid<\varepsilon$ for $x \in B\left(x_{0}, r\right) \cap \partial \Omega$. Then $f \leq f\left(x_{0}\right)+\varepsilon+2 M d / r$ on $\partial \Omega$. It follows that

$$
\limsup _{\Omega \ni y \rightarrow x_{0}} \bar{P} f(y) \leq f\left(x_{0}\right)+\varepsilon+\frac{2 M}{r} \lim _{\Omega \ni y \rightarrow x_{0}} \operatorname{Pd}(y)=f\left(x_{0}\right)+\varepsilon .
$$

Letting $\varepsilon \rightarrow 0$ gives $\lim \sup _{\Omega \ni y \rightarrow x_{0}} \bar{P} f(y) \leq f\left(x_{0}\right)$. Applying this to $-f$ we get

$$
\liminf _{\Omega \ni y \rightarrow x_{0}} \bar{P} f(y) \geq \liminf _{\Omega \ni y \rightarrow x_{0}} \underline{P} f(y)=-\limsup _{\Omega \ni y \rightarrow x_{0}} \bar{P}(-f)(y) \geq-\left(-f\left(x_{0}\right)\right)=f\left(x_{0}\right) .
$$

(e) $\Rightarrow$ (a) This follows directly from Definition 3.7, since continuous functions are resolutive by Theorem 3.5 .

(a) $\Rightarrow$ (c) Let us first consider the case when $C_{p}\left(\left\{x_{0}\right\}\right)=0$. Since $C_{p}(X \backslash \Omega)>0$, by assumption, we can find $B^{\prime}=B\left(x_{0}, r\right)$ so small that $C_{p}\left(X \backslash\left(\Omega \cup 2 B^{\prime}\right)\right)>0$. Let $u$ be the continuous solution of the $\mathscr{K}_{f, f}\left(\Omega \cup 2 B^{\prime}\right)$-obstacle problem, where $f(y)=-d\left(x_{0}, y\right)$. By Proposition 3.2, $\left.u\right|_{A}=H_{A} f$, where $A=\left\{y \in \Omega \cup 2 B^{\prime}: u(y)>f(y)\right\}$. 
It is clear that $u \leq 0$, that $u$ is bounded below and that $u\left(x_{0}\right)=0$. Let $G$ be a component of $A$, then $\left.u\right|_{G}=H_{G} f$. Since $C_{p}(X \backslash G) \geq C_{p}\left(X \backslash\left(\Omega \cup 2 B^{\prime}\right)\right)>0$, Lemma 4.3 shows that $C_{p}(\partial G)>0$. Since $C_{p}\left(\left\{x_{0}\right\}\right)=0$, it follows from the Kellogg property that there exists $x \in \partial G \backslash\left\{x_{0}\right\}$ which is regular for $G$. Hence $\lim _{G \ni y \rightarrow x} u(y)=f(x)<0$. Thus $u \not \equiv 0$ in $G$. By the strong maximum principle it follows that $u<0$ in $G$, and thus that $u<0$ in $\left(\Omega \cup 2 B^{\prime}\right) \backslash\left\{x_{0}\right\}$.

Let now $m=\sup _{\partial B^{\prime}} u<0$, by compactness. Since $\left.u\right|_{\left(\Omega \cup 2 B^{\prime}\right) \backslash \bar{B}^{\prime}}$ is the solution of the $\mathscr{K}_{f, u}\left(\left(\Omega \cup 2 B^{\prime}\right) \backslash \bar{B}^{\prime}\right)$-obstacle problem, we see that $\sup _{\left(\Omega \cup 2 B^{\prime}\right) \backslash \bar{B}^{\prime}} u=m$. It follows that $\lim \sup _{\Omega \ni y \rightarrow x} u(y) \leq m<0$ for all $x \in \partial \Omega \backslash \bar{B}^{\prime}$. Since $u$ is continuous in $2 B^{\prime}$, we also have $\lim \sup _{\Omega \ni y \rightarrow x} u(y)=u(x)<0$ for all $x \in\left(\partial \Omega \cap \bar{B}^{\prime}\right) \backslash\left\{x_{0}\right\}$, and $\lim _{\Omega \ni y \rightarrow x_{0}} u(y)=u\left(x_{0}\right)=0$.

Let now $v(x)=-\liminf _{\Omega \ni y \rightarrow x} u(y)$ for $x \in \partial \Omega$, and $w=\underline{P}_{\Omega} v$. Then $-u \in \mathscr{L}_{v}$ and hence $w \geq-u$. Thus,

$$
\liminf _{\Omega \ni y \rightarrow x} w(y) \geq-\limsup _{\Omega \ni y \rightarrow x} u(y)>0 \quad \text { for every } x \in \partial \Omega \backslash\left\{x_{0}\right\} .
$$

On the other hand, since $v$ is continuous at $x_{0}$ and bounded, the already proved implication $(\mathrm{a}) \Rightarrow(\mathrm{e})$ shows that $\lim _{\Omega \ni y \rightarrow x_{0}} w(y)=v\left(x_{0}\right)=0$. We have thus shown that $w$ is a positive $p$-harmonic barrier at $x_{0}$.

Let us finally consider the case when $C_{p}\left(\left\{x_{0}\right\}\right)>0$. Using Proposition 3.2, we let $u$ be the continuous solution of the $\mathscr{K}_{d, d}$-obstacle problem, and observe that $\left.u\right|_{A}=H_{A} d$, where $A=\{y \in \Omega: u(y)>d(y)\}$.

If $x_{0} \in \partial A$, then as $C_{p}\left(\left\{x_{0}\right\}\right)>0$, the Kellogg property implies that $x_{0}$ is regular for $A$, and hence $\lim _{A \ni y \rightarrow x_{0}} u(y)=d\left(x_{0}\right)=0$. On the other hand, if $x_{0} \in \partial(\Omega \backslash A)$, then $\lim _{\Omega \backslash A \ni y \rightarrow x_{0}} u(y)=\lim _{\Omega \backslash A \ni y \rightarrow x_{0}} d(y)=d\left(x_{0}\right)=0$. It follows that $\lim _{\Omega \ni y \rightarrow x_{0}} u(y)=0$ regardless of the location of $x_{0}$ on $\partial \Omega$. (Note that it is possible that $x_{0}$ belongs to both $\partial A$ and $\partial(\Omega \backslash A)$.) Moreover,

$$
\liminf _{\Omega \ni y \rightarrow x} u(y) \geq \liminf _{\Omega \ni y \rightarrow x} d(y)>0 \quad \text { for } x \in \partial \Omega \backslash\left\{x_{0}\right\}
$$

Since $u$ is $p$-superharmonic, $u$ is a positive continuous barrier at $x_{0}$.

(c) $\Rightarrow$ (b) This is trivial.

(b) $\Rightarrow$ (d) If $C_{p}\left(\left\{x_{0}\right\}\right)>0$, then $x_{0}$ is regular by the Kellogg property. Assume therefore that $C_{p}\left(\left\{x_{0}\right\}\right)=0$. Let $u$ be a barrier at $x_{0}$ and $G$ be a component of $\Omega$. Then $C_{p}(X \backslash G) \geq C_{p}(X \backslash \Omega)>0$, and by Lemma $4.3, C_{p}(\partial G)>0$. Thus there exists $x \in \partial G \backslash\left\{x_{0}\right\}$, and by the strong minimum principle $u>0$ in $G$. Since $u>0$ in every component, $u>0$ in $\Omega$.

Let $\varepsilon>0$ be so small that $\Omega^{\prime}:=\Omega \backslash \overline{B\left(x_{0}, \varepsilon\right)} \neq \varnothing$. Let $m=\inf _{\Omega^{\prime}} u$. We want to show that $m>0$. Let $\left\{y_{n}\right\}_{n=1}^{\infty}, y_{n} \in \Omega^{\prime}$, be a sequence such that $\lim _{n \rightarrow \infty} u\left(y_{n}\right)=m$. By compactness there is a convergent subsequence (also denoted $\left\{y_{n}\right\}_{n=1}^{\infty}$ ) with a limit $y_{0} \in \bar{\Omega}^{\prime}$. If $y_{0} \in \Omega$, then the lower semicontinuity of $u$ shows that $m \geq u\left(y_{0}\right)>0$. On the other hand if $y_{0} \in \partial \Omega$, then $m \geq \liminf _{\Omega \ni y \rightarrow y_{0}} u(y)>0$, since $y_{0} \neq x_{0}$. Thus $m>0$.

Let now $M=\sup _{\Omega} d$. Then $M u / m+\varepsilon \in \mathscr{U}_{d}$ and thus $P d \leq M u / m+\varepsilon$ from which it follows that 


$$
\limsup _{\Omega \ni y \rightarrow x_{0}} P d(y) \leq \frac{M}{m} \limsup _{\Omega \ni y \rightarrow x_{0}} u(y)+\varepsilon=\varepsilon
$$

Letting $\varepsilon \rightarrow 0$ shows that $\lim \sup _{\Omega \ni y \rightarrow x_{0}} \operatorname{Pd}(y) \leq 0$. That $\liminf _{\Omega \ni y \rightarrow x_{0}} \operatorname{Pd}(y) \geq 0$ is trivial.

Corollary 4.4. Let $x_{0} \in \partial \Omega$ be regular, and let $V \subset \Omega$ be open and such that $x_{0} \in \partial V$. Then $x_{0}$ is regular also with respect to $V$.

Proof. $\quad$ By Theorem 4.2, (a) $\Rightarrow(\mathrm{c})$, there is a positive continuous barrier $u$ at $x_{0}$ (with respect to $\Omega$ ). Let $v=\left.u\right|_{V}$. Then $\lim _{V \ni y \rightarrow x_{0}} v(y)=0$. For $x \in(\partial \Omega \cap \partial V) \backslash\left\{x_{0}\right\}$ we have $\liminf _{V \ni y \rightarrow x} v(y) \geq \liminf _{\Omega \ni y \rightarrow x} u(y)>0$. And for $x \in \partial V \backslash \partial \Omega$ we have $\lim _{V \ni y \rightarrow x} v(y)=u(x)>0$. Hence $v$ is a positive continuous barrier at $x_{0}$ with respect to $V$. It thus follows from Theorem $4.2,(\mathrm{c}) \Rightarrow(\mathrm{a})$, that $x_{0}$ is regular for $V$.

\section{Boundary regularity for the obstacle problem.}

The main result of this section is the following result.

TheOREm 5.1. Let $\psi: \Omega \rightarrow \overline{\boldsymbol{R}}$ and $f \in N^{1, p}(\Omega)$ be such that $\mathscr{K}_{\psi, f} \neq \varnothing$. Let $u$ be the lower semicontinuously regularized solution of the $\mathscr{K}_{\psi, f}$-obstacle problem. Let $x_{0} \in \partial \Omega$ be a regular boundary point. Assume further that either

(a) $f\left(x_{0}\right):=\lim _{\Omega \ni y \rightarrow x_{0}} f(y)$ exists, or

(b) $f \in N^{1, p}(\bar{\Omega} \cap B)$ for some ball $B$ centred at $x_{0}$, and that $\left.f\right|_{\partial \Omega \cap B}$ is continuous at $x_{0}$.

Then

$$
\lim _{\Omega \ni y \rightarrow x_{0}} u(y)=f\left(x_{0}\right)
$$

if and only if $f\left(x_{0}\right) \geq \operatorname{ess} \lim \sup _{\Omega \ni y \rightarrow x_{0}} \psi(y)$.

Note that it is possible to have $f\left(x_{0}\right)<\operatorname{ess} \lim \sup _{\Omega \ni y \rightarrow x_{0}} \psi(y)$ and still have a soluble obstacle problem, see Example 5.7 below.

The following corollary is a special case of Theorem 5.1.

Corollary 5.2. Let $f \in \operatorname{Lip}(\bar{\Omega})$, let $u$ be the continuous solution of the $\mathscr{K}_{f, f}$ obstacle problem and let $x_{0} \in \partial \Omega$ be regular. Then $\lim _{\Omega \ni y \rightarrow x_{0}} u(y)=f\left(x_{0}\right)$. In particular, if $\Omega$ is regular and we let $u=f$ on $\partial \Omega$, then $u \in C(\bar{\Omega})$.

Lemma 5.3. Let $u \in N^{1, p}(\Omega)$ and $v, w \in N_{0}^{1, p}(\Omega)$ be such that $v \leq u \leq w p$-q.e. in $\Omega$. Then $u \in N_{0}^{1, p}(\Omega)$.

Proof. By subtracting $v$ from all terms and observing that $u \in N_{0}^{1, p}(\Omega)$ if and only if $u-v \in N_{0}^{1, p}(\Omega)$, we may assume that $v \equiv 0$. After redefinitions on sets of $p$ capacity zero, we may, without loss of generality, assume that $0 \leq u \leq w$ everywhere in $\Omega$, and that $w=0$ in $X \backslash \Omega$.

Let $g_{u}^{\prime} \in L^{p}(\Omega)$ be an upper gradient of $u$ in $\Omega$, and let $g_{w}^{\prime} \in L^{p}(X)$ be an upper 
gradient of $w$ in $X$. Define

$$
\tilde{u}=\left\{\begin{array}{ll}
u, & \text { in } \Omega, \\
0, & \text { in } X \backslash \Omega,
\end{array} \text { and } g= \begin{cases}g_{u}^{\prime}+g_{w}^{\prime}, & \text { in } \Omega, \\
0, & \text { in } X \backslash \Omega .\end{cases}\right.
$$

We want to show that $g$ is an upper gradient of $\tilde{u}$, from which it follows that $\tilde{u} \in N^{1, p}(X)$ and thus $u \in N_{0}^{1, p}(\Omega)$. Let $\gamma:[a, b] \rightarrow X$ be a curve. If $\gamma([a, b]) \subset \Omega$, then

$$
|\tilde{u}(\gamma(a))-\tilde{u}(\gamma(b))|=|u(\gamma(a))-u(\gamma(b))| \leq \int_{\gamma} g_{u}^{\prime} d s \leq \int_{\gamma} g d s .
$$

On the other hand, if $\gamma(a), \gamma(b) \in X \backslash \Omega$, then $|\tilde{u}(\gamma(a))-\tilde{u}(\gamma(b))|=0 \leq \int_{\gamma} g d s$. By splitting $\gamma$ into two parts if necessary and possibly reversing the direction, we may thus assume that $\gamma(a) \in \Omega$ and $\gamma(b) \in X \backslash \Omega$. Let $c=\inf \{t \in[a, b]: \gamma(t) \in X \backslash \Omega\}$. By the continuity of $\gamma$, we have $\gamma(c) \in X \backslash \Omega$, i.e. $\tilde{u}(\gamma(b))=w(\gamma(c))=0$. Hence,

$$
|\tilde{u}(\gamma(a))-\tilde{u}(\gamma(b))|=|u(\gamma(a))| \leq|w(\gamma(a))-w(\gamma(c))| \leq \int_{\left.\gamma\right|_{(a, c)}} g_{w}^{\prime} d s \leq \int_{\gamma} g d s .
$$

LEMMA 5.4. Let $\psi_{j}: \Omega \rightarrow \overline{\boldsymbol{R}}$ and $f_{j} \in N^{1, p}(\Omega)$ be such that $\mathscr{K}_{\psi_{j}, f_{j}} \neq \varnothing$, and let $u_{j}$ be the lower semicontinuously regularized solution of the $\mathscr{K}_{\psi_{j}, f_{j}}$-obstacle problem, $j=1,2$. Assume that $\psi_{1} \leq \psi_{2} \mu$-a.e. in $\Omega$ and that $\left(f_{1}-f_{2}\right)_{+} \in N_{0}^{1, p}(\Omega)$, then $u_{1} \leq u_{2}$ in $\Omega$.

Proof. Let $u=\min \left\{u_{1}, u_{2}\right\}$ and $h=u_{1}-f_{1}-\left(u_{2}-f_{2}\right) \in N_{0}^{1, p}(\Omega)$. It follows that

$$
-\left(f_{2}-f_{1}\right)_{-}-h_{-} \leq \min \left\{f_{2}-f_{1}, h\right\} \leq h .
$$

By Lemma $5.3, \min \left\{f_{2}-f_{1}, h\right\} \in N_{0}^{1, p}(\Omega)$ and thus

$$
u-f_{1}=\min \left\{u_{2}-f_{1}, u_{1}-f_{1}\right\}=u_{2}-f_{2}+\min \left\{f_{2}-f_{1}, h\right\} \in N_{0}^{1, p}(\Omega) .
$$

Since $u \geq \psi_{1} \mu$-a.e. in $\Omega, u \in \mathscr{K}_{\psi_{1}, f_{1}}$.

Similarly $v=\max \left\{u_{1}, u_{2}\right\} \in \mathscr{K}_{\psi_{2}, f_{2}}$. Let $A=\left\{x \in \Omega: u_{1}(x)>u_{2}(x)\right\}$. Since $u_{2}$ is a solution of the $\mathscr{K}_{\psi_{2}, f_{2}}$-obstacle problem, we have

$$
\int_{\Omega} g_{u_{2}}^{p} d \mu \leq \int_{\Omega} g_{v}^{p} d \mu=\int_{A} g_{u_{1}}^{p} d \mu+\int_{\Omega \backslash A} g_{u_{2}}^{p} d \mu .
$$

Thus

$$
\int_{A} g_{u_{2}}^{p} d \mu \leq \int_{A} g_{u_{1}}^{p} d \mu
$$


It follows that

$$
\int_{\Omega} g_{u}^{p} d \mu=\int_{A} g_{u_{2}}^{p} d \mu+\int_{\Omega \backslash A} g_{u_{1}}^{p} d \mu \leq \int_{A} g_{u_{1}}^{p} d \mu+\int_{\Omega \backslash A} g_{u_{1}}^{p} d \mu=\int_{\Omega} g_{u_{1}}^{p} d \mu .
$$

Since $u_{1}$ is a solution of the $\mathscr{K}_{\psi_{1}, f_{1}}$-obstacle problem, so is $u$. By uniqueness $u_{1}=$ $u=\min \left\{u_{1}, u_{2}\right\}$ p-q.e. in $\Omega$, and thus $u_{1} \leq u_{2} p$-q.e. in $\Omega$. Since $u_{1}$ and $u_{2}$ are lower semicontinuously regularized it follows that $u_{1} \leq u_{2}$ everywhere in $\Omega$.

Lemma 5.5. Let $\psi: \Omega \rightarrow \overline{\boldsymbol{R}}$ and $f \in N^{1, p}(\Omega)$ be such that $\mathscr{K}_{\psi, f} \neq \varnothing$. Let $u$ be the lower semicontinuously regularized solution of the $\mathscr{K}_{\psi, f}$-obstacle problem and let $x_{0} \in \partial \Omega$. Assume that there exist a ball $B=B\left(x_{0}, r\right)$ and $k_{0} \in \boldsymbol{R}$ such that $\psi \leq k_{0}$ $\mu$-a.e. in $B \cap \Omega$ and $\left(f-k_{0}\right)_{+} \in N_{0}^{1, p}(\Omega ; B)$. Then for all $k \geq k_{0}$,

$$
\sup _{\Omega \cap \frac{1}{2} B} u \leq k+C\left(\frac{1}{\mu(B)} \int_{\Omega \cap B}(u-k)_{+}^{p} d \mu\right)^{1 / p}
$$

in particular $u$ is bounded from above in $\Omega \cap \frac{1}{2} B$.

Proof. Let $0<r_{1}<r_{2} \leq r$ and $k \geq k_{0}$ be arbitrary. Let $v=u-\eta(u-k)_{+}$, where $\eta \in \operatorname{Lip}_{c}\left(B\left(x_{0}, r_{2}\right)\right), 0 \leq \eta \leq 1, \eta=1$ in $B\left(x_{0}, r_{1}\right)$ and $g_{\eta} \leq C /\left(r_{2}-r_{1}\right)$. We shall show that $v \in \mathscr{K}_{\psi, f}$. Indeed, $\mu$-a.e. in $\Omega$ either $v=u \geq \psi$ or $v=(1-\eta) u+\eta k \geq \psi$. To show that $v-f \in N_{0}^{1, p}(\Omega)$, we observe that

$$
u-k=u-f+f-k_{0}-\left(k-k_{0}\right)
$$

and hence

$$
0 \leq \eta(u-k)_{+} \leq \eta(u-f)_{+}+\eta\left(f-k_{0}\right)_{+} \in N_{0}^{1, p}(\Omega \cap B) .
$$

Thus, by Lemma 5.3, $v-f=u-f-\eta(u-k)_{+} \in N_{0}^{1, p}(\Omega)$ and $v \in \mathscr{K}_{\psi, f}$.

Let $A_{j}=\left\{x \in B\left(x_{0}, r_{j}\right): u(x) \geq k\right\}, j=1,2$. We have $v=(1-\eta)(u-k)+k$ in $A_{2}$, and hence $g_{v} \leq(1-\eta) g_{u}+(u-k) g_{\eta} \mu$-a.e. in $A_{2}$. In $\Omega \backslash A_{2}$ we have $g_{v}=g_{u} \mu$-a.e. As $u$ is a solution of the $\mathscr{K}_{\psi, f}$-obstacle problem, we get

$$
\int_{A_{1}} g_{u}^{p} d \mu \leq \int_{A_{2}} g_{u}^{p} d \mu \leq \int_{A_{2}} g_{v}^{p} d \mu \leq C_{1} \int_{A_{2} \backslash A_{1}} g_{u}^{p} d \mu+C \int_{A_{2}}(u-k)^{p} g_{\eta}^{p} d \mu
$$

As $g_{\eta} \leq C /\left(r_{2}-r_{1}\right)$, adding $C_{1}$ times the left-hand side to both sides implies

$$
\int_{A_{1}} g_{u}^{p} d \mu \leq \theta \int_{A_{2}} g_{u}^{p} d \mu+\frac{C}{\left(r_{2}-r_{1}\right)^{p}} \int_{A_{2}}(u-k)^{p} d \mu
$$

where $\theta=C_{1} /\left(C_{1}+1\right)<1$. Lemma 3.1 in Chapter V in Giaquinta [11] then shows that for all $k \geq k_{0}$ and $0<r_{1}<r_{2} \leq r$, 


$$
\int_{A_{1}} g_{u}^{p} d \mu \leq \frac{C}{\left(r_{2}-r_{1}\right)^{p}} \int_{A_{2}}(u-k)^{p} d \mu .
$$

The lemma now follows from Theorem 4.3 in J. Björn [7] and the lower semicontinuity of $u$.

TheOrem 5.6. Let $\psi: \Omega \rightarrow \overline{\boldsymbol{R}}$ and $f \in N^{1, p}(\Omega)$ be such that $\mathscr{K}_{\psi, f} \neq \varnothing$. Let $u$ be the lower semicontinuously regularized solution of the $\mathscr{K}_{\psi, f}$-obstacle problem. Let $x_{0} \in \partial \Omega$ be a regular boundary point. Let

$$
\begin{aligned}
m & =\sup \left\{k \in \boldsymbol{R}:(f-k)_{-} \in N_{0}^{1, p}(\Omega ; B) \text { for some ball } B \text { centred at } x_{0}\right\}, \\
M^{\prime} & =\inf \left\{k \in \boldsymbol{R}:(f-k)_{+} \in N_{0}^{1, p}(\Omega ; B) \text { for some ball } B \text { centred at } x_{0}\right\}, \\
M & =\max \left\{M^{\prime}, \underset{\Omega \ni y \rightarrow x_{0}}{\left.\operatorname{ess} \limsup _{3} \psi(y)\right\} .}\right.
\end{aligned}
$$

Then

$$
m \leq \liminf _{\Omega \ni y \rightarrow x_{0}} u(y) \leq \limsup _{\Omega \ni y \rightarrow x_{0}} u(y) \leq M .
$$

ExAmple 5.7. (a) Note that it is not possible to replace $M$ by $M^{\prime}$. Let $\Omega=$ $(-1,1)^{n-1} \times(0,1), n \geq 2$,

$$
h\left(x^{\prime}, x_{n}\right)=\left\{\begin{array}{ll}
\frac{x_{n}}{\left|x^{\prime}\right|}, & 0<x_{n} \leq\left|x^{\prime}\right| \leq 1, \\
1, & \left|x^{\prime}\right|<x_{n} \leq 1, \\
0, & \text { otherwise, }
\end{array} \quad \eta(x)= \begin{cases}1, & |x| \leq \frac{1}{3} \\
2-3|x|, & \frac{1}{3} \leq|x| \leq \frac{2}{3} \\
0, & |x| \geq \frac{2}{3}\end{cases}\right.
$$

and $f=\eta h$, where $x=\left(x^{\prime}, x_{n}\right) \in \boldsymbol{R}^{n-1} \times \boldsymbol{R}$. After observing that $g_{h}=|\nabla h| \leq 2 /\left|x^{\prime}\right|$, it is straightforward to check that $f \in N^{1, p}\left(\boldsymbol{R}^{n}\right)$ for $1<p<n$. Hence $f \in \mathscr{K}_{f, f}$, and thus by Theorem 3.2 in Kinnunen-Martio [23], the $\mathscr{K}_{f, f}$-obstacle problem is soluble. In this case (with $x_{0}=0$ ) we have $m=M^{\prime}=0, M=1$ and

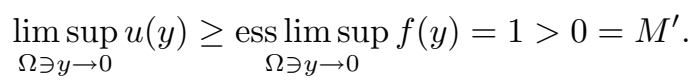

Let $a_{m}=1 / m$. By Theorem 5.6 (or Theorem 5.1),

$$
\lim _{\Omega \ni y \rightarrow\left(a_{m}, 0, \ldots, 0\right)} u(y)=f\left(a_{m}, 0, \ldots, 0\right)=0 .
$$

Hence there are $0<b_{m}<1 / m$ such that $u\left(a_{m}, 0, \ldots, 0, b_{m}\right)<1 / m$. It follows that

$$
\liminf _{\Omega \ni y \rightarrow 0} u(y) \leq \lim _{m \rightarrow \infty} u\left(a_{m}, 0, \ldots, 0, b_{m}\right)=0 .
$$


Since $0 \leq f \leq 1$ we know that $0 \leq u \leq 1$. And thus

$$
\liminf _{\Omega \ni y \rightarrow 0} u(y)=0 \quad \text { and } \quad \limsup _{\Omega \ni y \rightarrow 0} u(y)=1 .
$$

(b) If we let $f_{k}(x)=f(k x), k \geq 1$, then $\left\|f_{k}\right\|_{N^{1, p}\left(\boldsymbol{R}^{n}\right)}^{p} \leq k^{p-n}\|f\|_{N^{1, p}\left(\boldsymbol{R}^{n}\right)}^{p}$. It follows that $\tilde{f}=\sum_{j=0}^{\infty} f_{2^{j}} \in N^{1, p}\left(\boldsymbol{R}^{n}\right), 1<p<n$, and we get $m=M^{\prime}=0$ and $M=\infty$ (with respect to $\tilde{f}$ and $\left.x_{0}=0\right)$. In this case we get

$$
\liminf _{\Omega \ni y \rightarrow 0} u(y)=0 \quad \text { and } \quad \limsup _{\Omega \ni y \rightarrow 0} u(y)=\infty .
$$

Proof of Theorem 5.6. Let $k>M$ and find a ball $B=B\left(x_{0}, r\right)$ such that $(f-k)_{+} \in N_{0}^{1, p}(\Omega ; B)$ and $k \geq \operatorname{ess} \sup _{B \cap \Omega} \psi$. By Lemma $5.3,(u-k)_{+} \in N_{0}^{1, p}(\Omega ; B)$. Let $v=\max \{u, k\}=k+(u-k)_{+}$in $\Omega$ and $v=k$ on $B \backslash \Omega$. Then $v \in N^{1, p}(B)$. Let $G=\Omega \cap \frac{1}{3} B$ and $v^{\prime}=H_{G} v$. The minimum principle yields $v^{\prime} \geq k \geq \operatorname{ess} \sup _{G} \psi$ in $G$ and hence $v^{\prime}$ is a solution of the $\mathscr{K}_{\psi, v}(G)$-obstacle problem. Now, $u$ is clearly a solution of the $\mathscr{K}_{\psi, u}(G)$-obstacle problem and Lemma 5.4 shows that $u \leq v^{\prime}$ in $G$. As $\bar{G} \Subset B$ we can find $\eta \in \operatorname{Lip}_{c}(B)$ with $\eta=1$ on $\bar{G}$. It follows that $\eta v \in N^{1, p}(X)$ and thus $v^{\prime}=H_{G} v=H_{G} \eta v=P_{G} \eta v=P_{G} v$, by Theorem 3.6. Next, Lemma 5.5 shows that $v$ is bounded on $\bar{G}$. Corollary 4.4 shows that $x_{0}$ is regular also with respect to $G$. Hence, Theorem 4.2 (e) shows that

$$
\limsup _{\Omega \ni y \rightarrow x_{0}} u(y)=\limsup _{G \ni y \rightarrow x_{0}} u(y) \leq \lim _{G \ni y \rightarrow x_{0}} v^{\prime}(y)=v\left(x_{0}\right)=k
$$

Taking infimum over all $k>M$ shows one inequality of the theorem.

To prove the other inequality, note that $u \geq H f=-H(-f)$ and that $H(-f)$ is the lower semicontinuously regularized solution of the $\mathscr{K}_{-\infty,-f}$-obstacle problem. The first part of the proof applied to $-f$ with $M$ replaced by $-m$ then shows that

$$
\liminf _{\Omega \ni y \rightarrow x_{0}} u(y) \geq-\limsup _{\Omega \ni y \rightarrow x_{0}} H(-f)(y) \geq m
$$

Proof of Theorem 5.1. Assume first that $f\left(x_{0}\right) \geq \operatorname{ess} \lim \sup _{\Omega \ni y \rightarrow x_{0}} \psi(y)$, and let $m$ and $M$ be defined as in Theorem 5.6. Let $\varepsilon>0$, and let $B^{\prime}=B\left(x_{0}, r\right) \subset B$ be such that

$$
\left|f(x)-f\left(x_{0}\right)\right|<\varepsilon \text { for } \begin{cases}x \in B^{\prime} \cap \Omega & \text { in case (a), } \\ x \in B^{\prime} \cap \partial \Omega & \text { in case (b). }\end{cases}
$$

Then $\left(f-\left(f\left(x_{0}\right)+\varepsilon\right)\right)_{+} \in N_{0}^{1, p}\left(\Omega ; B^{\prime}\right)$. It follows that $M \leq f\left(x_{0}\right)+\varepsilon$ and letting $\varepsilon \rightarrow 0$ shows that $M \leq f\left(x_{0}\right)$. Similarly, $f\left(x_{0}\right) \leq m$, and by Theorem 5.6, $m \leq M$. Hence $\lim _{\Omega \ni y \rightarrow x_{0}} u(y)=m=M=f\left(x_{0}\right)$, by Theorem 5.6.

Conversely assume that $f\left(x_{0}\right)<\operatorname{ess} \lim \sup _{\Omega \ni y \rightarrow x_{0}} \psi(y)$. As $u \geq \psi \mu$-a.e., we see that 


$$
f\left(x_{0}\right)<\underset{\Omega \ni y \rightarrow x_{0}}{\operatorname{ess} \limsup } \psi(y) \leq \underset{\Omega \ni y \rightarrow x_{0}}{\operatorname{ess} \limsup } u(y) \leq \limsup _{\Omega \ni y \rightarrow x_{0}} u(y) .
$$

\section{Further characterizations of regularity.}

In this section we continue the characterization of regular boundary points from Theorem 4.2. The reason for splitting these equivalent conditions into two theorems is that Theorem 4.2 was needed to prove Corollary 4.4 and Theorems 5.1 and 5.6, which in turn are used in the proof of Theorem 6.1 below.

Theorem 6.1. Let $x_{0} \in \partial \Omega, \delta>0, B=B\left(x_{0}, \delta\right)$ and $d(x):=d\left(x, x_{0}\right)$. Then the following conditions are equivalent to the conditions in Theorem 4.2:

(f) The point $x_{0}$ is regular with respect to $G:=B \cap \Omega$.

(g) It is true that

$$
\lim _{\Omega \ni y \rightarrow x_{0}} H f(y)=f\left(x_{0}\right)
$$

for all $f \in N^{1, p}(\Omega)$ such that $f\left(x_{0}\right):=\lim _{\Omega \ni y \rightarrow x_{0}} f(y)$ exists.

(h) It is true that

$$
\lim _{\Omega \ni y \rightarrow x_{0}} H f(y)=f\left(x_{0}\right)
$$

for all $f \in N^{1, p}(\Omega \cup(B \cap \bar{\Omega}))$ such that $\left.f\right|_{\partial \Omega \cap B}$ is continuous at $x_{0}$.

(i) For all $f \in N^{1, p}(\Omega)$ and all $\psi: \Omega \rightarrow \overline{\boldsymbol{R}}$ such that $\mathscr{K}_{\psi, f} \neq \varnothing$ and

$$
f\left(x_{0}\right):=\lim _{\Omega \ni y \rightarrow x_{0}} f(y) \geq \underset{\Omega \ni y \rightarrow x_{0}}{\operatorname{ess} \limsup } \psi(y)
$$

(where the limit in the middle is assumed to exist), the lower semicontinuously regularized solution $u$ of the $\mathscr{K}_{\psi, f}$-obstacle problem satisfies

$$
\lim _{\Omega \ni y \rightarrow x_{0}} u(y)=f\left(x_{0}\right)
$$

(j) For all $f \in N^{1, p}(\Omega \cup(B \cap \bar{\Omega}))$ such that $\left.f\right|_{\partial \Omega \cap B}$ is continuous at $x_{0}$, and all $\psi: \Omega \rightarrow \overline{\boldsymbol{R}}$ such that $f\left(x_{0}\right) \geq \operatorname{ess} \lim \sup _{\Omega \ni y \rightarrow x_{0}} \psi(y)$ and $\mathscr{K}_{\psi, f} \neq \varnothing$, the lower semicontinuously regularized solution $u$ of the $\mathscr{K}_{\psi, f}$-obstacle problem satisfies

$$
\lim _{\Omega \ni y \rightarrow x_{0}} u(y)=f\left(x_{0}\right)
$$

(k) The continuous solution $u$ of the $\mathscr{K}_{d, d}$-obstacle problem satisfies

$$
\lim _{\Omega \ni y \rightarrow x_{0}} u(y)=0
$$


i.e. $u$ is a positive continuous barrier at $x_{0}$.

(l) For any function $f \in N^{1, p}(\bar{\Omega})$ which is $p$-superharmonic in $\Omega$ and such that $\left.f\right|_{\partial \Omega}$ is lower semicontinuous at $x_{0}$,

$$
\liminf _{\Omega \ni y \rightarrow x_{0}} f(y) \geq f\left(x_{0}\right)
$$

REMARKs 6.2. Condition (f) shows that regularity is a local property of the boundary.

Note that it is not possible to replace liminf by lim in (l) even if we require $\left.f\right|_{\partial \Omega}$ to be continuous at $x_{0}$, see Example 5.7.

The function $d$ in (d) and (k) can be replaced by any function $d^{\prime} \in C(\bar{\Omega}) \cap N^{1, p}(\Omega)$ with $d^{\prime}\left(x_{0}\right)=0$ and $d^{\prime}(x)>0$ for all $x \in \bar{\Omega} \backslash\left\{x_{0}\right\}$. In particular, we can have $d^{\prime}=d^{\alpha}$ with $\alpha>0$. In fact the following statements are true:

(A) Let $f$ be a bounded function on $\partial \Omega$ which is continuous at $x_{0}$ and such that

$$
\inf _{\partial \Omega \backslash B\left(x_{0}, r\right)} f>f\left(x_{0}\right) \quad \text { for all } r>0 .
$$

Then $x_{0}$ is regular if and only if

$$
\lim _{\Omega \ni y \rightarrow x_{0}} \underline{P} f(y)=f\left(x_{0}\right),
$$

which happens if and only if $\lim _{\Omega \ni y \rightarrow x_{0}} \bar{P} f(y)=f\left(x_{0}\right)$.

(B) Let $f \in N^{1, p}(\Omega)$ be such that $\lim _{\Omega \ni y \rightarrow x_{0}} f(y)=0$ and such that

$$
\inf _{\Omega \backslash B\left(x_{0}, r\right)} f>0 \quad \text { for all } r>0 .
$$

Then $x_{0}$ is regular if and only if

$$
\lim _{\Omega \ni y \rightarrow x_{0}} H f(y)=0 .
$$

(C) Let $f \in N^{1, p}(\Omega)$ be such that $\lim _{\Omega \ni y \rightarrow x_{0}} f(y)=0$ and $\psi: \Omega \rightarrow \overline{\boldsymbol{R}}$ be such that $\mathscr{K}_{\psi, f} \neq \varnothing$, ess $\lim \sup _{\Omega \ni y \rightarrow x_{0}} \psi(y) \leq 0$ and $\operatorname{ess} \liminf \inf _{\Omega \ni \rightarrow x} \psi(y)>0$ for $x \in \partial \Omega \backslash\left\{x_{0}\right\}$. Let $u$ be the lower semicontinuously regularized solution of the $\mathscr{K}_{\psi, f}$-obstacle. Then $x_{0}$ is regular if and only if

$$
\lim _{\Omega \ni y \rightarrow x_{0}} u(y)=0
$$

which happens if and only if $u$ is a barrier at $x_{0}$. (A similar statement can be based on (j) instead of (i).)

To prove this one can use Theorem 6.1 together with modifications of the proof of the implication $(\mathrm{d}) \Rightarrow(\mathrm{e})$. 
Proof. $\quad($ a) $\Rightarrow$ (i) and (a) $\Rightarrow$ (j) This follows from Theorem 5.1.

(i) $\Rightarrow(\mathrm{g})$ and $(\mathrm{j}) \Rightarrow(\mathrm{h})$ This is trivial as $H f$ is the continuous solution of the

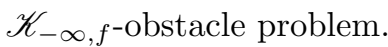

$(\mathrm{g}) \Rightarrow(\mathrm{d})$ and $(\mathrm{h}) \Rightarrow(\mathrm{d})$ This follows from Theorem 3.6, since $d \in N^{1, p}(X)$.

(i) $\Rightarrow(\mathrm{k})$ The first part is trivial. By Proposition 3.2, $u$ is continuous and $u \geq d$ everywhere in $\Omega$. Hence

$$
\liminf _{\Omega \ni y \rightarrow x} u(y) \geq d\left(x, x_{0}\right)>0 \quad \text { for all } x \in \partial \Omega \backslash\left\{x_{0}\right\} .
$$

Since $u$ is $p$-superharmonic, it is a positive continuous barrier.

$(\mathrm{k}) \Rightarrow(\mathrm{c})$ This is trivial.

(a) $\Rightarrow$ (f) This follows from Corollary 4.4 .

(f) $\Rightarrow$ (l) Let $\varepsilon>0$. Then there is $r \in(0, \delta)$ such that

$$
\inf _{B\left(x_{0}, r\right) \cap \partial G} f \geq f\left(x_{0}\right)-\varepsilon .
$$

Then $h:=\min \left\{f, f\left(x_{0}\right)-\varepsilon\right\}$ is $p$-superharmonic in $G$ and

$$
h-\left(f\left(x_{0}\right)-\varepsilon\right) \in N_{0}^{1, p}\left(G ; B\left(x_{0}, r\right)\right) .
$$

Since $h \in N^{1, p}(G)$, it is the lower semicontinuously regularized solution of the $\mathscr{K}_{h, h}(G)$ obstacle problem. We can therefore apply Theorem 5.6 (with $h$ and $G$ in the place of $f$ and $\Omega$ ). Observing that $m \geq f\left(x_{0}\right)-\varepsilon$, where $m$ is as in Theorem 5.6 , gives

$$
\liminf _{\Omega \ni y \rightarrow x_{0}} f(y)=\liminf _{G \ni y \rightarrow x_{0}} f(y) \geq \liminf _{G \ni y \rightarrow x_{0}} h(y) \geq f\left(x_{0}\right)-\varepsilon
$$

Letting $\varepsilon \rightarrow 0$ gives the desired conclusion.

$(\mathrm{l}) \Rightarrow(\mathrm{d})$ Let

$$
f= \begin{cases}H d, & \text { in } \Omega, \\ d, & \text { in } X \backslash \Omega .\end{cases}
$$

Then both $f$ and $-f$ satisfy the conditions in (l), and thus (d) follows, since $P d=H d$.

We end this section by discussing two further conditions on a boundary point.

(m) There is a weak barrier at $x_{0}$, i.e. a positive $p$-superharmonic function $u$ in $\Omega$ such that $\lim _{\Omega \ni y \rightarrow x_{0}} u(y)=0$.

(n) The point $x_{0}$ is regular with respect to every component $G \subset \Omega$ such that $x_{0} \in \partial G$.

The implication $(\mathrm{c}) \Rightarrow(\mathrm{m})$ is trivial and the implication $(\mathrm{a}) \Rightarrow(\mathrm{n})$ follows from Corollary 4.4. The implication $(\mathrm{n}) \Rightarrow(\mathrm{m})$ can be proved as follows:

$(\mathrm{n}) \Rightarrow(\mathrm{m})$ Let $G_{1}, G_{2}, \ldots$, be the components of $\Omega$ (either finitely or count- 
ably many). If $x_{0} \in \partial G_{j}$, then let $u_{j}$ be a barrier at $x_{0}$ with respect to $G_{j}$ and $u=\min \left\{u_{j}, 1 / j\right\}$ in $G_{j}$. If $x_{0} \notin \partial G_{j}$, then let $u \equiv 1 / j$ in $G_{j}$. Then $u: \Omega \rightarrow \boldsymbol{R}$ is a weak barrier at $x_{0}$.

The proof of $(\mathrm{n}) \Rightarrow(\mathrm{m})$ also shows that if $x_{0}$ has a neighbourhood $B=B\left(x_{0}, r\right)$, $r>0$, such that every $x \in \bar{B}, x \neq x_{0}$, is contained in the boundary of at most finitely many components of $\Omega$, then there is a (strong) barrier at $x_{0}$ with respect to $\Omega \cap B$, and thus (a) holds (after using that (a) $\Leftrightarrow$ (f) $\Leftrightarrow$ (b)).

In the classical linear setting (unweighted $\boldsymbol{R}^{n}$ with $p=2$ ) it is true that $(\mathrm{m}) \Rightarrow(\mathrm{b})$, see, e.g., Doob [10], Section 1.VIII.12, or Armitage-Gardiner [1], Lemma 6.6.3. Thus (a)-(n) are all equivalent in the classical linear setting.

Conjecture 6.3. Condition (m) (and hence also (n)) is equivalent to (a)-(l).

\section{Pointwise estimates at the boundary.}

In this section, we give a pointwise estimate near the boundary for solutions of the obstacle problem in linearly locally connected spaces. It is a generalization of a similar estimate for $p$-harmonic functions from Theorem 5.1 in Björn-MacManusShanmugalingam [8]. This estimate implies the sufficiency part of the Wiener criterion for regularity of boundary points, see Corollary 7.3 and Remark 7.4.

Definition 7.1. We say that $X$ is linearly locally connected if there exist $C_{0}>0$ and $r_{0}>0$ such that for all balls $B$ in $X$ with radius at most $r_{0}$, every pair of points in the annulus $2 B \backslash \bar{B}$ can be connected by a curve lying in the annulus $2 C_{0} B \backslash C_{0}^{-1} \bar{B}$.

Proposition 4.5 in Hajłasz-Koskela [12] shows that every complete pathconnected $Q$ regular metric space satisfying a $(1, Q)$-Poincaré inequality is linearly locally connected. In our pointwise estimate we use the relative capacity

$$
\operatorname{Cap}_{p}(E, 2 B)=\inf _{v} \int_{2 B} g_{v}^{p} d \mu
$$

where the infimum is taken over all $v \in N_{0}^{1, p}(2 B)$ satisfying $v \geq 1$ on $E \subset \bar{B}$. By Lemma 3.3 in J. Björn [7], the capacities $\operatorname{Cap}_{p}$ and $C_{p}$ have the same zero sets and are in many cases equivalent. In particular,

$$
\frac{C_{p}(E)}{C\left(1+r^{p}\right)} \leq \operatorname{Cap}_{p}(E, 2 B) \leq 2^{p-1}\left(1+\frac{1}{r^{p}}\right) C_{p}(E)
$$

where $r$ is the radius of $B$. To simplify the notation, we let

$$
W(\rho, r)=\int_{\rho}^{r}\left(\frac{\operatorname{Cap}_{p}\left(B\left(x_{0}, t\right) \backslash \Omega, B\left(x_{0}, 2 t\right)\right)}{\operatorname{Cap}_{p}\left(B\left(x_{0}, t\right), B\left(x_{0}, 2 t\right)\right)}\right)^{1 /(p-1)} \frac{d t}{t}
$$

TheOREM 7.2. Assume that $X$ is linearly locally connected with constants $C_{0}$ and $r_{0}$. Let $f \in N^{1, p}(\bar{\Omega})$ and let $\psi: \Omega \rightarrow \boldsymbol{R}$ be essentially bounded from above and such that 
$\mathscr{K}_{\psi, f} \neq \varnothing$. Let $u$ be the lower semicontinuously regularized solution of the $\mathscr{K}_{\psi, f \text {-obstacle }}$ problem. Let $x_{0} \in \partial \Omega$ and assume that $\left.f\right|_{\partial \Omega}$ is continuous at $x_{0}$ and that $f\left(x_{0}\right)=0$ (for simplicity). Let $0<\rho \leq r \leq r_{0} / 2 C_{0}$ and $B=B\left(x_{0}, 5 C_{0}^{2} r\right)$. Then

$$
\begin{aligned}
& \sup _{\Omega \cap B\left(x_{0}, \rho\right)} u \leq \max \left\{\sup _{\partial \Omega \cap 2 B} f, \operatorname{ess~sup}_{\Omega \cap 2 B} \psi\right\}+\max \left\{\sup _{\partial \Omega} f, \underset{\Omega}{\operatorname{ess} \sup \psi\} \exp (-C W(\rho, r)),}\right. \\
& \inf _{\Omega \cap B\left(x_{0}, \rho\right)} u \geq \inf _{\partial \Omega \cap 2 B} f+\inf _{\partial \Omega} f \exp (-C W(\rho, r)),
\end{aligned}
$$

where $C>0$ depends only on $X$.

We have the freedom to change $f$ on a set of $p$-capacity zero without changing $u$. Such a change may improve the estimates in the theorem.

Proof. $\quad$ Let $G=\Omega \cap B, K=\overline{B\left(x_{0}, r\right)} \backslash \Omega$,

$$
k=\max \left\{\sup _{\partial \Omega \cap 2 B} f, \operatorname{ess~sup}_{\Omega \cap 2 B} \psi\right\}
$$

and $h=k+\left(\sup _{\Omega} u-k\right) h^{\prime}$, where $h^{\prime}$ is the $p$-harmonic function in $B \backslash K$ with boundary values 0 on $\partial K$ and 1 on $\partial B$. By the Kellogg property we have $h \geq u$ p-q.e. on $\partial G$, and Lemma 5.4 shows that $u \leq h$ in $G$. The capacitary estimate for $h^{\prime}$ from Lemma 5.7 in Björn-MacManus-Shanmugalingam [8] together with the estimate

$$
\sup _{\Omega} u \leq \max \left\{\sup _{\partial \Omega} f, \underset{\Omega}{\operatorname{ess} \sup } \psi\right\}
$$

proves the first inequality.

The second inequality follows from the first inequality as in the proof of Theorem 5.6: $u \geq H f=-H(-f)$ and $H(-f)$ is the lower semicontinuously regularized solution of the

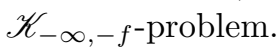

Corollary 7.3 (Sufficiency of the Wiener criterion). Assume that $X$ is linearly locally connected and $x_{0} \in \partial \Omega$. If $W(0, r)=\infty$ for some $r>0$, then $x_{0}$ is regular.

Proof. Let $0<\rho \leq \varepsilon \leq \min \left\{r, r_{0} / 2 C_{0}\right\}$. Theorem 7.2 with $f(x)=d(x)=$ $d\left(x, x_{0}\right)$ and $\psi=-\infty$ implies

$$
\sup _{\Omega \cap B\left(x_{0}, \rho\right)} H d \leq 10 C_{0}^{2} \varepsilon+(\operatorname{diam} \Omega) \exp (-C W(\rho, \varepsilon)) .
$$

As $W(\varepsilon, r) \leq \int_{\varepsilon}^{r} t^{-1} d t=\log (r / \varepsilon)<\infty$, we have $W(0, \varepsilon)=W(0, r)-W(\varepsilon, r)=\infty$, and hence $\lim _{\rho \rightarrow 0+} W(\rho, \varepsilon)=\infty$. Thus for sufficiently small $\rho$,

$$
\sup _{\Omega \cap B\left(x_{0}, \rho\right)} H d \leq 20 C_{0}^{2} \varepsilon
$$


and letting $\varepsilon \rightarrow 0$ together with Theorem 6.1 , (d) $\Rightarrow$ (a), finishes the proof.

REMARK 7.4. Instead of Theorem 7.2 in the proof of Corollary 7.3, we could have used Theorem 5.1 from [8]. It is formulated for boundary data $f \in N^{1, p}(X) \cap C(\bar{\Omega})$ and therefore does not directly imply Corollary 7.3. However, together with our Theorem 6.1 or Theorem 3.9 in Björn-Björn-Shanmugalingam [4] (which shows that a boundary point is regular with respect to $f \in C(\partial \Omega)$ if and only if it is regular with respect to Lipschitz boundary data) it can be used to prove Corollary 7.3.

\section{References}

[1] D. H. Armitage and S. J. Gardiner, Classical Potential Theory, Springer-Verlag, London, 2001.

[2] A. Björn, Characterizations of $p$-superharmonic functions on metric spaces, Studia Math., 169 (2005), 45-62.

[ 3 ] A. Björn and J. Björn, Boundary regularity for $p$-harmonic functions and solutions of the obstacle problem, Preprint, LiTH-MAT-R-2004-09, Linköping, 2004.

[4] A. Björn, J. Björn and N. Shanmugalingam, The Dirichlet problem for $p$-harmonic functions on metric spaces, J. Reine Angew. Math., 556 (2003), 173-203.

[5] A. Björn, J. Björn and N. Shanmugalingam, The Perron method for $p$-harmonic functions, J. Differential Equations, 195 (2003), 398-429.

[6] A. Björn, J. Björn and N. Shanmugalingam, Quasicontinuity of Newton-Sobolev functions and density of Lipschitz functions on metric spaces, Preprint, LiTH-MAT-R-2006-01, Linköping, 2006.

[ 7 ] J. Björn, Boundary continuity for quasiminimizers on metric spaces, Illinois J. Math., 46 (2002), 383-403.

[8] J. Björn, P. MacManus and N. Shanmugalingam, Fat sets and pointwise boundary estimates for p-harmonic functions in metric spaces, J. Anal. Math., 85 (2001), 339-369.

[9] G. Dal Maso, U. Mosco and M. A. Vivaldi, A pointwise regularity theory for the two-obstacle problem, Acta Math., 163 (1989), 57-107.

[10] J. L. Doob, Classical Potential Theory and its Probabilistic Counterpart, Springer-Verlag, New York, 1984

[11] M. Giaquinta, Multiple Integrals in the Calculus of Variations and Nonlinear Elliptic Systems, Princeton Univ. Press, Princeton, N. J., 1983.

[12] P. Hajłasz and P. Koskela, Sobolev met Poincaré, Mem. Amer. Math. Soc., 145 (2000).

[13] J. Heinonen, Lectures on Analysis on Metric Spaces, Springer-Verlag, New York, 2001.

[14] J. Heinonen, T. Kilpeläinen and O. Martio, Nonlinear Potential Theory of Degenerate Elliptic Equations, Oxford Univ. Press, Oxford, 1993.

[15] J. Heinonen, P. Koskela, N. Shanmugalingam and J. Tyson, Sobolev Spaces on Metric Measure Spaces: an Approach Based on Upper Gradients, In preparation.

[16] S. Keith, Modulus and the Poincaré inequality on metric measure spaces, Math. Z., 245 (2003), $255-292$.

[17] S. Keith and X. Zhong, The Poincaré inequality is an open ended condition, Preprint, Jyväskylä, 2003.

[18] T. Kilpeläinen, J. Kinnunen and O. Martio, Sobolev spaces with zero boundary values on metric spaces, Potential Anal., 12 (2000), 233-247.

[19] T. Kilpeläinen and J. Malý, The Wiener test and potential estimates for quasilinear elliptic equations, Acta Math., 172 (1994), 137-161.

[20] T. Kilpeläinen and W. P. Ziemer, Pointwise regularity of solutions to nonlinear double obstacle problems, Ark. Mat., 29 (1991), 83-106.

[21] J. Kinnunen and O. Martio, The Sobolev capacity on metric spaces, Ann. Acad. Sci. Fenn. Math., 21 (1996), 367-382.

[22] J. Kinnunen and O. Martio, Choquet property for the Sobolev capacity in metric spaces, in Proceedings on Analysis and Geometry (Novosibirsk, Akademgorodok, 1999), Sobolev Institute Press, Novosibirsk, 2000, pp. 285-290.

[23] J. Kinnunen and O. Martio, Nonlinear potential theory on metric spaces, Illinois Math. J., 46 
(2002), 857-883.

[24] J. Kinnunen and N. Shanmugalingam, Regularity of quasi-minimizers on metric spaces, Manuscripta Math., 105 (2001), 401-423.

[25] P. Koskela and P. MacManus, Quasiconformal mappings and Sobolev spaces, Studia Math., 131 (1998), 1-17.

[26] T. Laakso, Ahlfors $Q$-regular spaces with arbitrary $Q>1$ admitting weak Poincaré inequality, Geom. Funct. Anal., 10 (2000), 111-123.

[27] H. Lebesgue, Conditions de régularité, conditions d'irrégularité, conditions d'impossibilité dans le probème de Dirichlet, C. R. Acad. Sci. Paris, 178 (1924), 349-354.

[28] V. G. Maz'ya, On the continuity at a boundary point of solutions of quasi-linear elliptic equations, Vestnik Leningrad. Univ. Mat. Mekh. Astronom., 25:13 (1970), 42-55 (Russian). English transl.: Vestnik Leningrad Univ. Math., 3 (1976), 225-242.

[29] P. Mikkonen, On the Wolff potential and quasilinear elliptic equations involving measures, Ann. Acad. Sci. Fenn. Math. Dissertationes, 104 (1996).

[30] L. Saloff-Coste, Aspects of Sobolev-type Inequalities, London Math. Soc. Lecture Note Ser., 289, Cambridge Univ. Press, Cambridge, 2002.

[31] N. Shanmugalingam, Newtonian spaces: An extension of Sobolev spaces to metric measure spaces, Rev. Mat. Iberoamericana, 16 (2000), 243-279.

[32] N. Shanmugalingam, Harmonic functions on metric spaces, Illinois J. Math., 45 (2001), 10211050 .

[33] N. Shanmugalingam, Some convergence results for $p$-harmonic functions on metric measure spaces, Proc. London Math. Soc., 87 (2003), 226-246.

[34] N. Shanmugalingam, Private communication, 2003.

[35] N. Wiener, The Dirichlet problem, J. Math. Phys., 3 (1924), 127-146.

\section{Anders BJÖRN}

Department of Mathematics

Linköpings universitet

SE-581 83 Linköping

Sweden

E-mail: anbjo@mai.liu.se

\section{Jana BJÖRN}

Department of Mathematics Linköpings universitet SE-581 83 Linköping

Sweden

E-mail: jabjo@mai.liu.se 